\title{
Targeting of Locus Ceruleus Noradrenergic Neurons Expressing Human Interleukin-2 Receptor $\alpha$-Subunit in Transgenic Mice by a Recombinant Immunotoxin anti-Tac(Fv)-PE38: A Study for Exploring Noradrenergic Influence upon Anxiety-Like and Depression-Like Behaviors
}

\author{
Keiichi Itoi, ${ }^{1,2}$ Naoya Sugimoto, ${ }^{1}$ Saya Suzuki, ${ }^{1,2}$ Keisuke Sawada, ${ }^{1}$ Gopal Das, ${ }^{1}$ Katsuya Uchida, ${ }^{1}$ Toshimitsu Fuse, ${ }^{1}$ \\ Shinji Ohara, ${ }^{3}$ and Kazuto Kobayashi ${ }^{4}$ \\ ${ }^{1}$ Laboratory of Information Biology, Graduate School of Information Sciences, and ${ }^{2}$ Division of Neuroendocrinology, Graduate School of Medicine, Tohoku \\ University, Sendai 980-8579, Japan, 32Department of Neurology, Matsumoto Medical Center, Matsumoto 399-0021, Japan, and 4Department of Molecular \\ Genetics, Institute of Biomedical Sciences, Fukushima Medical University, Fukushima 960-1295, Japan
}

The noradrenergic (NA) neurons in the locus ceruleus (LC) were ablated with a high degree of selectivity by immunotoxin-mediated neuronal targeting. Transgenic mice were used in which the human interleukin-2 receptor- $\alpha$ subunit (hIL-2R $\alpha$; Tac) is expressed under the promoter of dopamine $\beta$-hydroxylase. The recombinant immunotoxin, which is composed of the Fv fragment of an anti-hIL-2R $\alpha$ monoclonal antibody fused to a truncated form of Pseudomonas exotoxin [anti-Tac(Fv)-PE38], was injected bilaterally into the LC of the mouse. As a result, the LC-NA neurons disappeared almost completely, and tissue noradrenaline was depleted in brain regions that receive NA inputs from the LC. The decrement of tissue noradrenaline content was more profound compared with that in mice treated with $\mathrm{N}$-(2-chloroethyl)- $\mathrm{N}$-ethyl-2-bromobenzylamine (DSP-4), a neurotoxin capable of ablating axons originating from the LC-NA neurons. Mice treated with either the immunotoxin or DSP-4 presented increased anxiety-like behaviors; in contrast, only the immunotoxintreated mice, and not the DSP-4-treated mice, showed increased depression-like behavior. The immunotoxin-mediated neuronal targeting may provide a means for further unraveling the links between the LC and pathological manifestations of neurological disorders.

\begin{abstract}
Introduction
The locus ceruleus (LC) is the largest noradrenergic (NA) nucleus in the brain and sends out axon terminals to most brain regions. The LC has been implicated in sleep/wakefulness (González and Aston-Jones, 2006), attention and alertness (Berridge and Waterhouse, 2003; Aston-Jones et al., 2007), and anxiety and depression (Brunello et al., 2003), as well as cognitive processes (Murchison et al., 2004; Sterpenich et al., 2006). Although degenerative loss of LC-NA neurons, in addition to nigrostriatal dopaminergic neurons, is marked in Parkinson's disease (Benarroch, 2009), it is not clear whether the defective NA system is related to part of the diverse
\end{abstract}

Received Oct. 3, 2010; revised Feb. 7, 2011; accepted March 1, 2011.

Author contributions: K.I. and N.S. designed research; K.I., N.S., S.S., K.S., G.D., K.U., T.F., and S.O. performed research; K.K. contributed unpublished reagents/analytic tools; K.I., N.S., S.S., and K.S. analyzed data; K.I., T.F., and K.K. wrote the paper.

K.I. and N.S. contributed equally to this work.

We thank Dr. Ira Pastan, Laboratory of Molecular Biology, National Cancer Institute, National Institutes of Health, for providing us with the immunotoxin. We thank Dr. Tatsushi Onaka, Jichi Medical University, Dr. Yasunobu Yasoshima, Osaka University, and Dr. Eiko Sakurai, Iwaki Meisei University, for technical advice. This work is supported in part by research grants from JSPS (to K.I.) and JST (CREST) (to K.K.).

Correspondence should be addressed to Dr. Keiichi Itoi, Laboratory of Information Biology, Graduate School of Information Sciences, Tohoku University, Sendai 980-8579, Japan. E-mail: itoik@m.tohoku.ac.jp.

DOI:10.1523/JNEUROSCI.5188-10.2011

Copyright $\odot 2011$ the authors $\quad 0270-6474 / 11 / 316132-08 \$ 15.00 / 0$ spectrum of parkinsonian symptoms, including anxiety and depression (Martínez-Martín and Damián, 2010).

In previous examinations of the role of the LC, two neurotoxins, $N$-(2-chloroethyl)- $N$-ethyl-2-bromobenzylamine (DSP-4) and 6-hydroxydopamine (6-OHDA) have been used to selectively disrupt the LC-NA neurons. However, there are methodological disadvantages associated with the use of these neurotoxins. First, site variability is unavoidable even following careful microinjection of 6-OHDA into specific brain regions (Gaillet et al., 1993). Second, axons originating from the medullary NA nuclei, i.e., the nucleus of the solitary tract (NTS) and ventrolateral reticular formation (VLR), may also be affected since 6-OHDA is readily taken up by nerve endings and axons en passage (Engelbrecht et al., 1994). DSP-4 destroys the NA terminals originating from the LC with considerable selectivity following systemic administration (Jonsson et al., 1981; Fritschy and Grzanna, 1989). However, the majority of the cell bodies of the LC-NA neurons are intact for weeks despite substantial loss of NA nerve endings in the projection fields. In addition, regeneration of axon terminals follows within months (Fritschy and Grzanna, 1992). The dopamine $\beta$-hydroxylase (DBH) knock-out mouse is an efficient model (Murchison et al., 2004), but all NA neurons, including those in the LC, NTS, and VLR, are disabled 
without spatial selectivity. Therefore, it is not possible to attribute any functional defect observed in this model to any specific NA cell group(s) in the brain.

The immunotoxin-mediated neuronal targeting is a strategy to ablate a selective population of neurons by application of the immunotoxins into a brain region of genetically engineered animals (Kobayashi et al., 1995). The recombinant immunotoxin used in the present study is a chimeric protein composed of a truncated form of Pseudomonas exotoxin and a single-chain Fv fragment of anti-hIL-2R $\alpha$ monoclonal antibody (anti-Tac) (Chaudhary et al., 1989; Kreitman et al., 1994) and recognizes the heterologous protein, hIL-2R $\alpha$, expressed in a selective population of neurons. After binding to hIL-2R $\alpha$ and being internalized into the cytoplasm, it triggers arrest of protein synthesis, resulting in elimination of the targeted cells (Keppler-Hafkemeyer et al., 1998). In the present study, transgenic mice were used, in which the hIL-2R $\alpha$ is expressed under the promoter of the gene encoding $\mathrm{DBH}$, an enzyme to catalyze synthesis of noradrenaline. The LC-NA neurons were ablated selectively and thoroughly following stereotaxic delivery of the immunotoxin bilaterally into the LC of the transgenic mice. Behavioral tests were performed to explore the relationship between the loss of LC-NA neurons and anxiety or depression.

\section{Materials and Methods}

\section{Animals}

All animal procedures were approved by the Committee on Use and Care of Animals, Tohoku University. Transgenic mice that express hIL-2R $\alpha$ under the control of the DBH promoter were used (Kobayashi et al., 1995). Heterozygous male and female mice (9-10 weeks of age) were used. They had been backcrossed over 10 generations with C57BL/6N mice. Mice were housed in groups before surgery, allowed ad libitum access to food and water, and maintained on a $12 \mathrm{~h}$ light/dark cycle (lights on, 07:00-19:00). DNA was prepared from individual tails at 4 weeks of age and subjected to PCR analysis (Kobayashi et al., 1995).

\section{Surgery and treatment}

Under intraperitoneal (i.p.) chloral hydrate ( $400 \mathrm{mg} / \mathrm{kg}$ body weight) anesthesia, a pair of holes was made in the occipital bones stereotaxically, and a tapered glass pipette was inserted into the LC. The coordinates were as follows: $5.3 \mathrm{~mm}$ caudal to the bregma, $\pm 0.9 \mathrm{~mm}$ lateral to the midline, and 3.6 $\mathrm{mm}$ ventral from the surface of the cerebellum. The recombinant immunotoxin, anti-Tac(Fv)-PE38 (Kreitman et al., 1994), was dissolved in PBS at pH 7.5 to a final concentration of $24 \mu \mathrm{g} / \mathrm{ml}$. The toxin solution or PBS (a total of $0.2 \mu \mathrm{l}$ ) was injected at the rate of $0.05 \mu \mathrm{l} / \mathrm{min}$. After surgery, each mouse was housed in an individual cage for 3-28 d, depending on the experimental protocol. Mice were handled daily for a week until the day before the behavioral experiments. The PBS-injected transgenic mice served as the control for the histological and behavioral studies. Wild-type C57BL/6N mice were used in control studies for the immunotoxin-induced ablation of the LC-NA neurons as well as in studies for DSP-4 treatment. Following i.p. injection of DSP-4 (50 mg/kg), mice were housed in individual cages. Naive mice (both transgenic and wild-type) were also used as controls in the biochemical and histological time course studies.

\section{Immunohistochemistry}

For the time course study of immunohistochemistry, mice were deeply anesthetized and perfused transcardially with $0.9 \%$ saline and $4 \%$ paraformaldehyde at either $3,7,10,14$, or $28 \mathrm{~d}$ after surgery. Naive mice were also treated in the same manner for evaluating the morphology at the basal state. Thirty-micrometer cryosections were stained with antityrosine hydroxylase (TH; rabbit, 1:1000, Millipore Bioscience Research Reagents) and/or anti-hIL-2R $\alpha$ antibody (mouse, 1:100, Dako). The mouse corpus striatum was stained with the anti-TH antibody which served as a positive control. By Western blot, the antibody labeled a single band at $\sim 62 \mathrm{kDa}$, which corresponded to TH according to the manufacturer's data sheet. Cy3-conjugated anti-rabbit IgG (Millipore Bioscience
Research Reagents) and Alexa488-conjugated anti-mouse IgG (Invitrogen) were used as secondary antibodies. A confocal laser scanning microscope (Leica TCS 4PI) was used for the analysis of colocalization of hIL-2R $\alpha$ with TH. Using sections obtained from each brain region $(n=$ 6 for the LC-ablated or the control group), the density of TH-ir varicosities was scored by two expert researchers (K.I. and S.S.) independently.

\section{Biochemical assay}

The tissue catecholamine contents were analyzed in mice treated with either the immunotoxin or DSP-4 and compared with those in respective controls ( $n=4-11$ for each group). Mice were decapitated under i.p. chloral hydrate anesthesia at 7, 10, 14, or $28 \mathrm{~d}$ following treatment with either the immunotoxin or its vehicle, and their brains were removed quickly. Following treatment with either DSP-4 or its vehicle, mice were decapitated at day 28. Naive mice, without treatment, were processed in the same manner. Each brain region was dissected out from 2-mm-thick slices, put in a plastic tube, frozen in liquid nitrogen, and weighed. The tissue sample was homogenized in $0.1 \mathrm{~m}$ perchloric acid containing $5 \mathrm{~mm}$ EDTA2Na and $0.1 \mathrm{~mm}$ sodium hydrogen sulfite and centrifuged at $15,000 \times g$. After filtering, the concentrations of noradrenaline, dopamine, and serotonin were measured by high performance liquid chromatography coupled with electrochemical detection (Yanai et al., 1998).

\section{Behavioral experiments}

Behavioral experiments were conducted 3 weeks following surgery because it took $\sim 2$ weeks for the tissue noradrenaline content to reach its lowest point (see Results). Only male mice were used for behavioral studies to prevent possible variations of the behaviors depending on the estrous cycle in females. The elevated plus-maze (EPM), open-field test, light-dark box, and forced swim were performed between 08:00 and 15:00 as a consecutive $4 \mathrm{~d}$ session. The behavior was monitored in the next room via a video camera apparatus. All test sessions were recorded, and videotapes were subsequently scored by trained observers blind to the group assignment (K.I., N.S., and K.S.). After the behavioral tests, histological examinations were made in the pontine region to verify the successful LC ablation in every immunotoxin-injected transgenic mouse. Biochemical measurements were made in the hippocampal tissue for every immunotoxin-injected transgenic mouse and DSP-4treated mouse to verify the successful depletion of noradrenaline. The hippocampus was chosen because it is one of the regions which receive NA inputs solely from the LC, and thus, the hippocampal tissue noradrenaline content reflects the amount of NA projections originating from the LC.

EPM. The plus-maze consisted of two open arms $(27 \mathrm{~cm} \times 5 \mathrm{~cm})$ and two closed arms $(27 \mathrm{~cm} \times 5 \mathrm{~cm})$ placed $38.5 \mathrm{~cm}$ above the ground. The closed arms were concealed by $15-\mathrm{cm}$-high walls. A mouse was placed in the center square $(5 \mathrm{~cm} \times 5 \mathrm{~cm})$ facing a closed arm and allowed to explore the maze for $5 \mathrm{~min}$ in a dimly lit room $(40 \mathrm{~lx})$.

Open-field test. The open field was a brightly lit (140 lx), white, open arena $(42 \mathrm{~cm} \times 42 \mathrm{~cm})$ concealed by $30-\mathrm{cm}$-high walls. After placement of a mouse in the center of the box, the ambulatory behavior was monitored for $10 \mathrm{~min}$. The field was divided into nine squares; the scored measures were the number of crossings between squares, the number of entry into the central square, and the time spent in the central square.

Light-dark box. The apparatus comprised two boxes, a light one (21 $\mathrm{cm} \times 30 \mathrm{~cm} \times 21 \mathrm{~cm}, 540 \mathrm{~lx})$ and a dark one $(21 \mathrm{~cm} \times 15 \mathrm{~cm} \times 21 \mathrm{~cm})$, and each box was connected by a hemicircular tunnel. Mice were placed in the dark box, and three parameters, i.e., the latency to coming out into the light box, the time spent in the light box, and the number of transitions, were counted for $10 \mathrm{~min}$.

Forced swim test. Mice were placed in a Plexiglas cylinder $(25 \mathrm{~cm}$ height and $10 \mathrm{~cm}$ internal diameter) containing tepid water $\left(25^{\circ} \mathrm{C}\right)$ to the height of $15 \mathrm{~cm}$. An 8 min test duration was used. Total duration of immobility was scored from videotape during the last $6 \mathrm{~min}$ of the test period. The water was changed between subjects.

\section{Statistics}

All data were expressed as means \pm SEM. Kolmogorov-Smirnov test was used to check the normality of the samples. Time course data were analyzed by ANOVA, followed by post hoc Sheffe $F$ test. Biochemical data 
were analyzed by unpaired Student's $t$ test, and behavioral data by Mann-Whitney $U$ test. $p$ values of $<0.05$ were regarded as statistically significant. All statistics were performed using computer software (IBM SPSS Statistics 19 and Stat View, Abacus Concepts).

\section{Results}

Topography of the hIL-2R $\alpha$ expression with reference to LC-NA neurons

The hIL-2R $\alpha$ immunoreactivity (ir) was expressed in anatomically identified NA neurons including those in the LC (Fig. 1a) in the transgenic mouse. Aberrant expression of the hIL-2R $\alpha$-ir was limited to a small number of cells. A quantitative analysis was made in the LC using 20 sections from six transgenic mice, and the $\mathrm{TH}$-immunoreactive neurons were all $(100.0 \pm 0.0 \%)$ immunoreactive for hIL- $2 \mathrm{R} \alpha$, and $98.6 \pm 0.6 \%$ of the hIL$2 \mathrm{R} \alpha$-immunoreactive neurons were $\mathrm{TH}$ immunoreactive. The TH-ir was present not only in the NA perikarya but also in the axonal radiation arising from the LC (Fig. 1a). However, the hIL-2R $\alpha$-ir was difficult to detect in axonal projections of the NA neurons despite its conspicuous expression within the cell bodies (and part of the dendrites) of the LC-NA neurons (Fig. 1a).

\section{Histological and biochemical} examinations following ablation of the LC-NA neurons

Within $10 \mathrm{~d}$ following bilateral immunotoxin injection into the $\mathrm{LC}$ of a transgenic mouse, almost all cell bodies and axons of the LC-NA neurons disappeared (Fig. 1b). The immunotoxin had no effect on the LC-NA neurons when it was injected into the wild-type animals (Fig. 1b, supplemental Fig. S1, available at www.jneurosci.org as supplemental material). The locus subceruleus (A5), the ventral continuum of the LC, was ablated in the same manner as the principal LC in the transgenic mouse (Fig. 1c), but it was not affected in wild-type animals (Fig. 1c). The number of LC-NA cell bodies decreased considerably by day 3 , were mostly gone by day 7 , and completely disappeared by day 10 without any recovery on day 28 (Fig. 1d, supplemental Fig. S1). The sham operation had no effects on the morphology of the LC-NA neurons in either the transgenic mice or wild-type mice from day 3 through day 28 after surgery (supplemental Fig. S1). Histological examinations were performed up to 3 months following immunotoxin injection, and no regeneration of the LC-NA neurons could be identified (data not shown).

Histological examination revealed a marked decrease in the intensity of TH-immunoreactive axon terminals in the cerebral

d
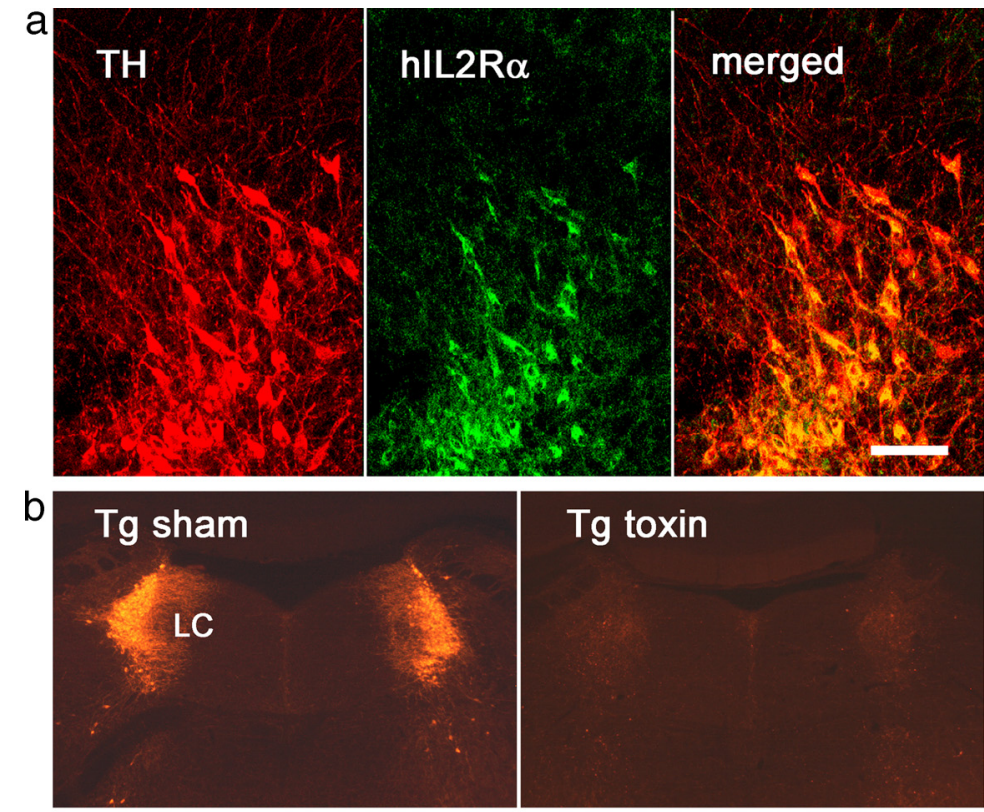

$\mathrm{Tg}$ toxin
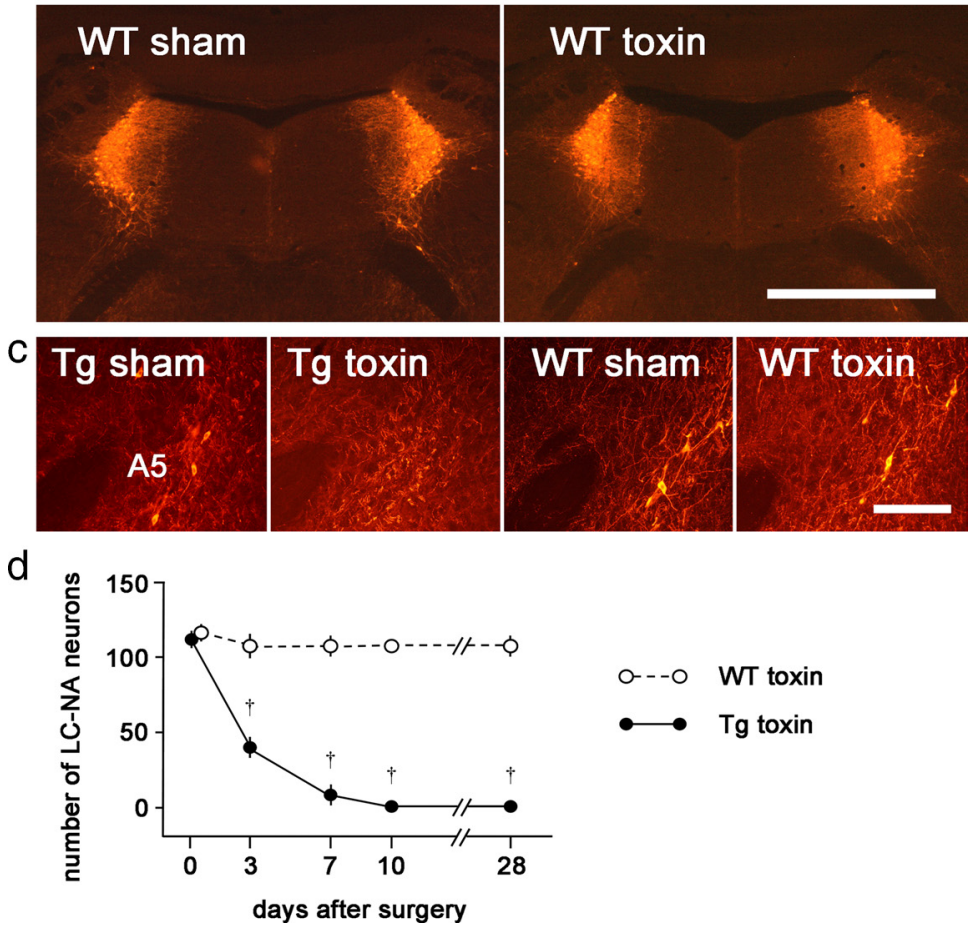

Figure 1. Disruption of the LC-NA neurons by the immunotoxin. $\boldsymbol{a}$, The colocalization of the hIL-2R $\alpha$ in the LC-NA neurons of a transgenic $(\mathrm{Tg})$ mouse was examined by confocal microscopy. The horizontal sections of the $\mathrm{LC}$ of the Tg mouse were stained with either the TH or the hlL-2R $\alpha$ antibody. The TH-immunoreactive (NA) neurons expressed the hlL-2R $\alpha$-ir, and vice versa. $\boldsymbol{b}$, By reference to the control ( $\mathrm{Tg}$ sham) animals, TH-immunoreactive $\mathrm{LC}$ neurons disappeared completely $10 \mathrm{~d}$ following immunotoxin injection bilaterally into the LC (Tg toxin). The immunotoxin had no effect on the morphology of LC-NA neurons in the wild-type mice (WT toxin) compared with the sham-operated controls (WT sham). c, The A5 NA cells were also ablated by immunotoxin treatment in Tg animals (Tg toxin) compared with the control (Tg sham), but not in wild-type mice (WT toxin) compared with sham-operated controls (WT sham). $\boldsymbol{d}$, Following immunotoxin injection, the number of TH-immunoreactive cell bodies was counted per side for the transgenic (Tg toxin, closed circle) and wild-type (WT toxin, open circle) mice, and compared with that in mice without treatment (day 0 ). The number of LC-NA neurons decreased considerably by day 3 , were mostly gone by day 7 , and completely disappeared by day 10 , without any recovery at day 28 . Sections containing the highest number of cells were selected for each animal. Means \pm SEM. $n=6$ for each group. ${ }^{\dagger} p<0.01$ vs time $=0$. Scale bars: $\boldsymbol{a}, \boldsymbol{c}, 100 \mu \mathrm{m} ; \boldsymbol{b}, 1.0 \mathrm{~mm} ; \mathrm{A} 5, \mathrm{~A} 5 \mathrm{NA}$ cell group.

cortex, hippocampus, thalamic nuclei (with the exception of the paraventricular nucleus of the thalamus [PVT]), and cerebellum, as well as in some discrete areas in the pontine and medullary regions following immunotoxin injection (Table 1, Fig. $2 a, b$ ). In 
Table 1. Comparison of the density of TH-immunoreactive axon terminals between the control and LC-NA-ablated mice

\begin{tabular}{lll}
\hline & TH-ir axon density & \\
\cline { 2 - 3 } Brain region & Control $(n=6)$ & LC-ablated $(n=6)$ \\
\hline PO & & \\
MPO & + & + \\
Hypothalamus & & +++ \\
PVH & ++ & ++ \\
SON & ++ & \\
Amygdala & & ++ \\
CeA & ++ & ++ \\
BLA & ++ & \\
Thalamus & & +++ \\
PVT & +++ & \pm \\
AV & ++ & \pm \\
VLT & ++ & \\
Cortex & & \pm \\
Cg & ++ & \pm \\
SM & ++ & \pm \\
Hippocampus & & \\
DG/CA1-3 & + & \pm \\
Cerebellum & & + \\
Mol/Gran & + & ++ \\
Pons & & \\
Pr5 & ++ & \\
VC & ++ & \\
Medulla & ++ & \\
Sp5 & ++ & \\
10N & & \\
\hline
\end{tabular}

Ten days following immunotoxin injection into bilateral LC, density of the TH-immunoreactive axon terminals was scored from + to +++ , the latter being the highest. \pm , Undefined or only very sparsely observed. PBS was injected in the control mice. MPO, medial preoptic area; $S O N$, supraoptic nucleus; CeA, central amygdaloid nucleus; BLA, basolateral amygdaloid nucleus; PVT, paraventricular nucleus of thalamus; $\mathrm{AV}$, anteroventral thalamic nucleus; VLT, ventrolateral thalamic nucleus; $C g$, cingulate cortex; SM, sensorimotor cortex; DG, dentate gyrus; Mol, molecular layer; Gran, granular layer; $\mathrm{Pr} 5$, principle trigeminal sensory nucleus; $\mathrm{VC}$, ventral cochlear nucleus; Sp5, spinal trigeminal nucleus; $10 \mathrm{~N}$, dorsal motor nucleus of vagus.

the LC-ablated mice, the tissue content of noradrenaline decreased significantly (Figs. $2 c, 3 a$ ). The decrement was $\sim 40 \%$ at day $7,60 \%$ at day 10 , and $90 \%$ at day 14 in the hippocampus (Fig. $2 c$ ), one of the regions in which noradrenaline decreased most profoundly. The noradrenaline content did not recover in these regions at 4 weeks following immunotoxin injection (Fig. 2c). At this time, the tissue noradrenaline also decreased significantly in the cerebellum, cortex, medulla, thalamus, midbrain, amygdaloid region, and preoptic area (PO)/nucleus accumbens (Acb) (Fig. $3 a$ ). In the hypothalamus, however, it was not significantly different between the LC-ablated and control mice (Fig. 3a). The density of $\mathrm{TH}$-immunoreactive nerve endings was not significantly different between the groups in major hypothalamic nuclei including the PVH and supraoptic nucleus (Table 1). The tissue noradrenaline content did not decrease either in the wild-type mice treated with immunotoxin or its vehicle or in sham-operated transgenic mice (supplemental Fig. S2, available at www.jneurosci.org as supplemental material).

Despite the thorough disruption of the LC-NA neurons, the NA cell bodies and axons were perfectly spared in the NTS (A2) (Fig. $2 d$ ) and the VLR (A1) (Fig. 2e), the two major NA nuclei in the medulla oblongata. The number of $\mathrm{TH}$-immunoreactive neurons was not significantly different between the control and the LC-ablated group in either of these nuclei: in the A2, $18.2 \pm$ 1.4 and $18.7 \pm 1.0$ (per section, single side, $n=10$ ) for the control and LC-ablated mice, respectively; and in the A1, 19.2 \pm 0.7 and $20.9 \pm 1.2(n=10)$, respectively. A considerable number of $\mathrm{TH}$-immunoreactive neurons in the $\mathrm{A} 7$, located in the rostrolateral vicinity of the $\mathrm{LC} /$ subceruleus complex, also decreased following the LC ablation (data not shown).
In the DSP-4-treated mice, the decrement of tissue noradrenaline content at 4 weeks was less profound than that in the immunotoxin-injected mice, although the overall regional profile was similar to that in the immunotoxin-treated group (Fig. $3 a$ ). In the hypothalamus, the noradrenaline content decreased slightly but significantly in the DSP-4-treated mice. The tissue dopamine content was not significantly affected in brain regions receiving mesostriatal dopaminergic projections in either the immunotoxin-treated or the DSP-4-treated group (Fig. 3b). Increased dopamine content was observed in the amygdaloid region in both the immunotoxin-treated-and DSP-4-treated mice, the increase being more significant in the immunotoxin-treated group. The tissue serotonin content did not differ between groups, with the exception of a slight increase in the amygdaloid region in the immunotoxin-treated group (Fig. $3 c$ ).

\section{Effects of ablation of the LC-NA neurons on animal behavior EPM}

The time spent in the open arm $(p<0.01)$, the number of entries into the open arms $(p<0.01)$, and the percentage of open arm entries $(p<0.05)$ were significantly smaller in mice whose LC-NA neurons were ablated with immunotoxin $(n=21)$ compared with those in sham-operated mice $(n=16)$ (Fig. $4 a)$. The total number of entries into closed arms, as a measure for locomotor activity, was not significantly different between the groups (Fig. 4a).

\section{Open-field test}

The time spent in the central square, the number of entries into the central square, and the percentage of central square entries were significantly smaller in mice with immunotoxin-induced ablation of the LC-NA neurons $(n=20)$ compared with those in sham-operated mice $(n=16)(p<0.05)$ (Fig. $4 b)$. The total number of crossings was not significantly different between the groups (Fig. 4b).

\section{Light-dark box}

The number of transitions was significantly smaller in mice whose LC-NA neurons were ablated by immunotoxin $(n=20)$ compared with that in sham-operated mice $(n=16)(p<0.05)$ (Fig. $4 c$ ). The latency to entry into the light compartment was longer, and the time spent in the light box was smaller, in the immunotoxin group, although the group differences were not statistically significant (Fig. 4c).

Across the anxiety battery, the behavioral parameters in the wild-type mice with bilateral immunotoxin injection into the LC $(n=14)$ were equivalent to those in the sham-operated (vehicleinjected) wild-type mice $(n=14)$. Hence, the observed changes could not be due to possible nonspecific effects of the immunotoxin (supplemental Fig. S3, available at www.jneurosci.org as supplemental material).

With respect to the DSP-4-treated mice $(n=13)$, the profiles of the anxiety-related behaviors resembled those in the immunotoxin-treated mice, but the observed values were significantly different from those in controls $(n=15)$ in only some of the behavioral assessments (Fig. $5 a-c$ ): in the EPM, the behaviors of DSP-4-treated mice were not significantly different from those in controls (Fig. $5 a$ ). In the open-field test, the number and percentage of central square entries were significantly decreased compared with control mice $(p<0.05)$ (Fig. $5 b)$, but the time spent in the central square did not differ significantly. In the light-dark box, the time spent in the light box was significantly smaller in the DSP-4-treated mice $(p<0.01)$, whereas the latency to appear in the light box and the number of transitions were not 
significantly different between the DSP-4treated mice and the controls (Fig. 5c).

Forced swim test

The immobilization time was significantly greater $(p<0.001)$ in mice with immunotoxin-induced ablation of LC-NA neurons $(n=20)$ compared with that in the sham-operated mice $(n=15)$ (Fig. 6a). However, it was not significantly different in DSP-4-treated mice $(n=13)$ compared with the vehicle-treated controls $(n=15)$ (Fig. 6b).

The immobilization time in the wildtype mice with bilateral immunotoxin injection into the LC $(n=14)$ was equivalent to that in the sham-operated (vehicle-injected) wild-type mice ( $n=$ 14). Hence, the observed changes could not be due to possible nonspecific effects of the immunotoxin (supplemental Fig. S3).

\section{Discussion}

With the use of the immunotoxinmediated neuronal targeting, the LC-NA neurons were ablated in a spatially selective manner. The cell bodies of the LC-NA neurons were eliminated within $10 \mathrm{~d}$ following injection of the immunotoxin, and no morphological or biochemical signs of regeneration were observed after 1 month. The ablated NA cell bodies were confined to the LC and its continuum. The medullary NA nuclei, the A1 and A2, were totally spared, demonstrating the spatial selectivity of this method. Since the expression of the hIL-2R $\alpha$-ir was not observed in the axons of the NA neurons despite its conspicuous expression in the cell bodies, the bulk of immunotoxins may not have been taken up by the axon terminals to undergo retrograde axonal transport. This is probably the reason for the completely intact NA nuclei in the medulla, which send out axons to the LC-NA neurons.

The spatial selectivity of the method was further validated in the projection fields of the LC-NA neurons in the context of previous findings obtained in the rat. Following ablation of the NA cell bodies in the LC, a remarkable decrease in the tissue noradrenaline content was observed in brain regions including the cerebellum, hippocampus, cerebral cortex, medulla, thalamus, midbrain, amygdaloid region, and PO/Acb. However, there was a striking difference, between these brain regions, in the degree of decrement of the NA projections following ablation of the LC-NA cell bodies in biochemical and histological examinations. The decrease was most prominent $(>90 \%)$ in the cortex, hippocampus, and cerebellum, in which the vast majority of the NA inputs were reported to originate from the LC in the rat (Moore and Card, 1984). In the medulla, thalamus, midbrain, and amygdala, the tissue noradrenaline content decreased by $\sim 50-75 \%$. In the
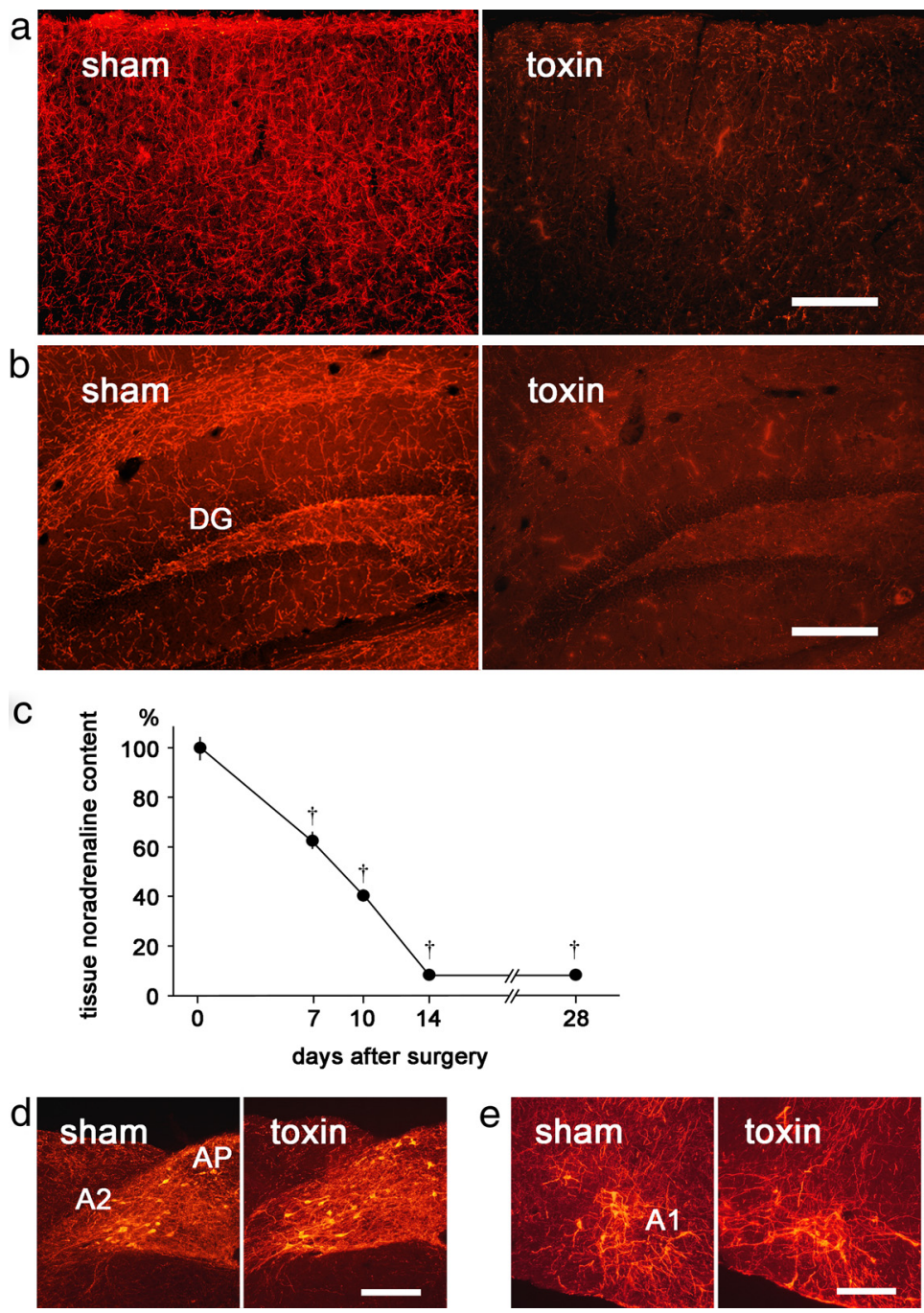

Figure 2. Significant effects of the immunotoxin-induced ablation of LC-NA neurons on the NA-containing axon terminals in the cortex and hippocampus, but not on the NA cell bodies in the medulla. $\boldsymbol{a}$, Compared with the TH-immunoreactive nerve endings mmunotoxin injection into the Tg mice (toxin). $\boldsymbol{b}$, In the hippocampus, TH-immunoreactive nerve endings also decreased in the mmunotoxin-treated Tg animals (toxin) compared with those in sham-operated Tg animals (sham). c, In the hippocampus, it took 4 weeks. Naive Tg mice without treatment were used for reference (day 0 ). Means \pm SEM. $n=6-11 .{ }^{\dagger} p<0.01$ vs time $=0$. The number and shape of the TH-immunoreactive neurons were not affected in the A2 NA nuclei following immunotoxin y immunotoxin injection to the Tg mice (toxin) in comparison with those in the sham-operated Tg mice (sham). Scale bars: $\boldsymbol{a}, \boldsymbol{b}$ $\boldsymbol{d}, \boldsymbol{e}, 200 \mu \mathrm{m}$. DG, dentate gyrus; AP, area postrema; A2, A2 NA cell group in the NTS; A1, A1 NA cell group in the VLR.

rat, these regions receive NA inputs from both the $\mathrm{LC}$ and the lateral tegmentum (Moore and Card, 1984), which may also explain the present results in the mouse. In contrast, the decrease was much less prominent in the PO/Acb area $(\sim 30 \%)$, and no significant decrease was observed in the hypothalamus. This may reflect the significantly greater contribution of the medullary NA nuclei as the source of NA inputs to these regions. Indeed, the hypothalamic and preoptic areas receive major NA inputs from the medullary NA nuclei in the rat (Palkovits, 1981; Cunningham and Sawchenko, 1988; Fritschy and Grzanna, 1989; Aston-Jones et al., 1999) although there is also a certain amount of inputs from the LC (Palkovits, 1981; Cunningham and Sawchenko, 1988; Aston-Jones et al., 1999; España and Berridge, 2006). Histological studies were made in multiple reference regions in the present study, and overall, the results were correlated with the biochem- 


\section{a tissue noradrenaline content}
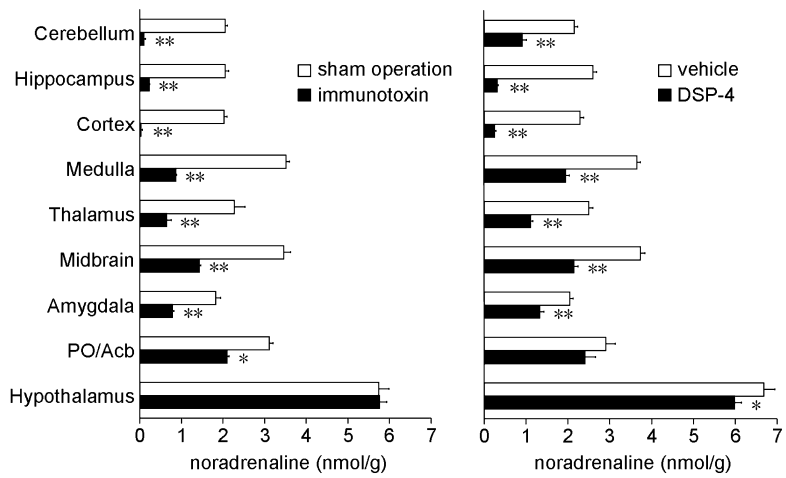

b tissue dopamine content
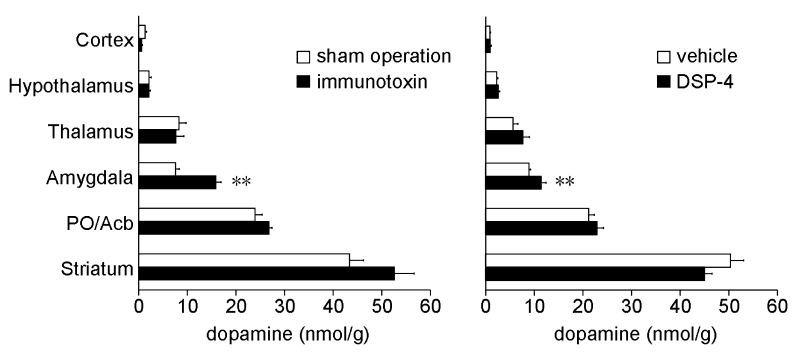

C tissue serotonin content
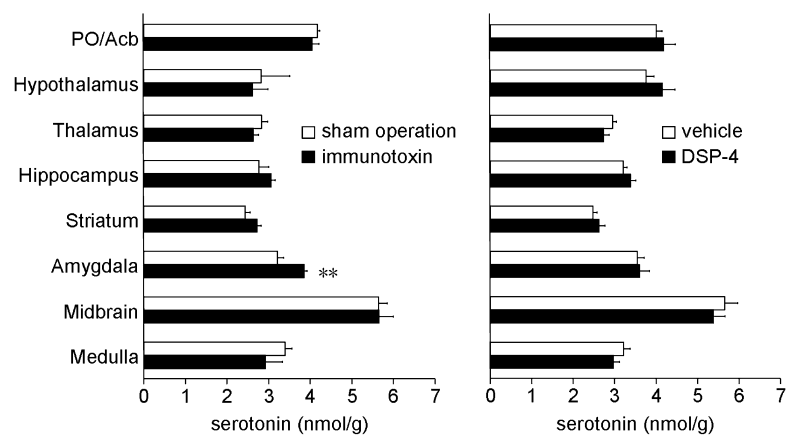

Figure 3. Noradrenaline, dopamine, and serotonin tissue content in brain regions. Regional profiles of tissue catecholamine contents are shown at 4 weeks following immunotoxin (left panel) and DSP-4 (right panel) treatment. The tissue noradrenaline content decreased in all brain regions except for the hypothalamus following immunotoxin injection compared with that in control ( $\boldsymbol{a}$, left panel). The decrease was most prominent in the cerebellum, hippocampus, and cortex. The noradrenaline content decreased in a similar profile after DSP-4 injection $(\boldsymbol{a}$, right panel), but the decrease was less conspicuous. Neither the dopamine content $(\boldsymbol{b})$ nor serotonin content (c) changed significantly following immunotoxin or DSP-4 injection, with the exception of the amygdala (dopamine increased both in the immunotoxin-treated group and DSP-4treated group, while serotonin increased only in the immunotoxin-treated group). If the values in the vehicle-treated animals are $<2 \mathrm{nmol} / \mathrm{g}$, data are not shown. $n=4-7$ for each group. Means \pm SEM. ${ }^{*} p<0.05 ;{ }^{* *} p<0.01$ vs respective control group. P0, Preoptic area; Acb, nucleus accumbens.

ical data. There are some distinct subdivisions or nuclei which were revealed to be innervated almost exclusively by the LC (or conversely by the medullary nuclei). In the thalamus, for example, most thalamic nuclei became devoid of the $\mathrm{TH}$ immunoreactive nerve endings following ablation of LC-NA neurons, but only the PVT was not affected at all, consistent with the reported innervation pattern of the thalamus in the rat (Moore and Card, 1984). Other examples of anatomically defined nuclei, which were revealed to receive major innervation from the LC, are the principal trigeminal sensory nucleus and cochlear nuclei (the pontine region) and spinal trigeminal nucleus (the medullary region). Thus, the distribution of NA nerve endings in the mouse was similar to that in the rat, which has been a elevated plus-maze
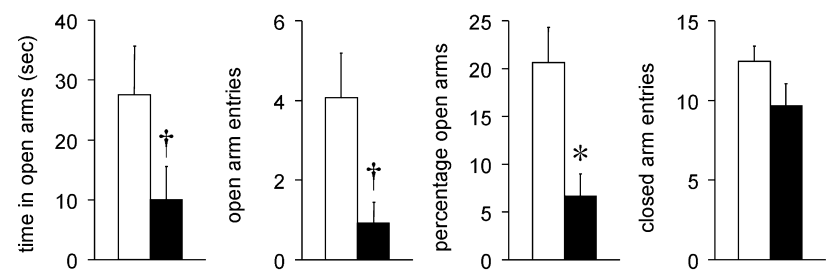

b open field
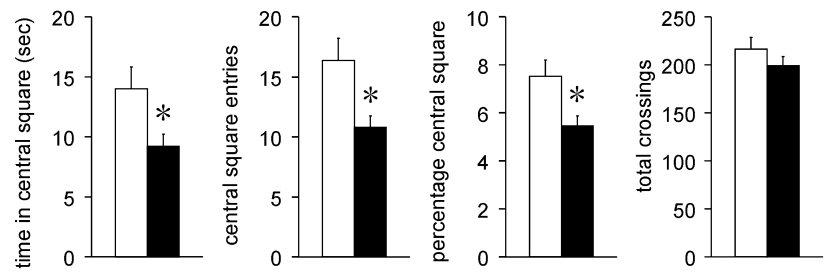

\section{C light-dark box}
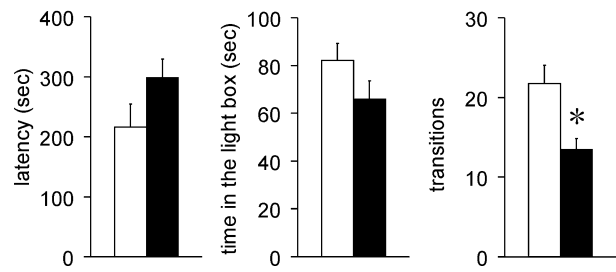

\section{$\square$ control \\ immunotoxin}

Figure 4. Anxiety-like behaviors in animals whose LC-NA neurons were ablated by the immunotoxin. $\boldsymbol{a}$, In the EPM, the time spent in the open arm, the number of open arm entries, and percentages of open arm entries were significantly smaller in the LC-NA-ablated mice $(n=21)$ compared with the control $(n=16)$. The number of entries into the closed arms was not different between groups. $\boldsymbol{b}$, In the open-field test, the time spent in the central square and the number of entries into the central square, as well as the percentage of entry into the central square, were smaller significantly in the LC-NA-ablated mice $(n=20)$ compared with the control ( $n=16)$. The total number of crossings between squares was not different between groups. $c$, In the light-dark box, the number of transitions was significantly smaller in the LC-NA neuron-ablated mice $(n=20)$ than in controls $(n=16)$. The latency was longer and the time spent in the light box was smaller in the former group, but these values were not statistically significant. Means \pm SEM. ${ }^{*} p<0.05 ;{ }^{\dagger} p<0.01$ vs sham-operated (control) mice.

made clear by ablation of the NA nuclei (Palkovits, 1981; Cunningham and Sawchenko, 1988), transection of their axonal tracts (Kromer and Moore, 1980; Palkovits, 1981), or retrograde and anterograde tracings (Cunningham and Sawchenko, 1988; Aston-Jones et al., 1999; España and Berridge, 2006).

Most of the cell bodies of LC-NA neurons disappeared by day 7 following immunotoxin injection, whereas the tissue noradrenaline content decreased by only $40 \%$ at day 7 , by $60 \%$ at day 10 , and reached the lowest level at day 14 . Thus, there was an intriguing discrepancy in the time course between the degeneration of NA cell bodies in the LC and the tissue noradrenaline content in the projection fields. The mechanism for the delayed disappearance of tissue noradrenaline is not clear, but it may be related to the "anterograde" nature of axonal degeneration in the present method. For example, it was at 2 weeks following electrolytic ablation of the LC (anterograde degeneration) that the $\sim 90 \%$ decrement of tissue noradrenaline content was observed in the cortex (Levitt and Moore, 1979). In contrast, the tissue noradrenaline content was reportedly reduced by $80-90 \%$ in the cortex and hippocampus within 1 week following treatment with DSP-4, which is a "retrograde" neurotoxin (Logue et al., 1985; our unpublished observation). Once the maximal noradrenaline 
a elevated plus-maze
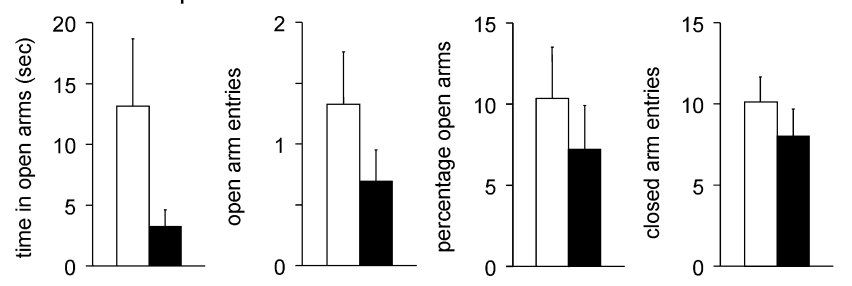

b open field
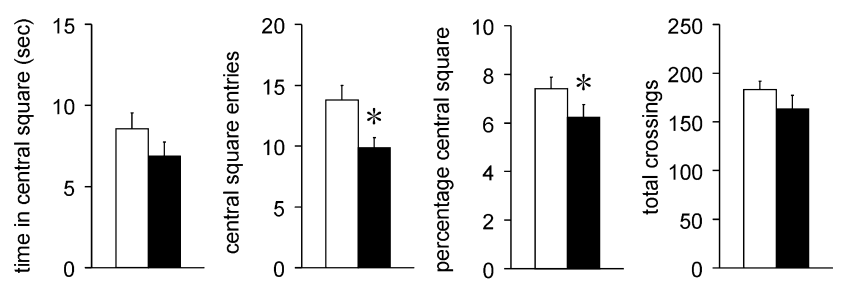

\section{C light-dark box}
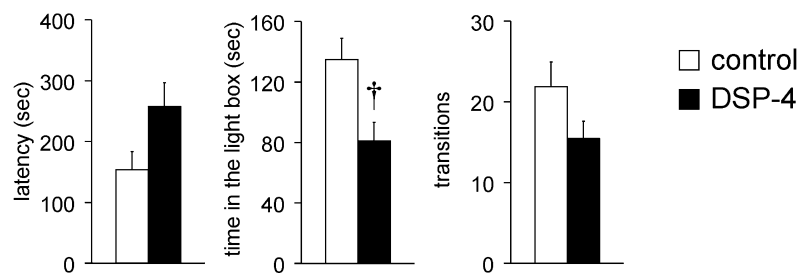

DSP-4

Figure 5. Anxiety-like behaviors in animals whose axon terminals of the LC-NA neurons were ablated by DSP-4. $\boldsymbol{a}$, In the EPM, the anxiety-like behaviors were not different significantly between the DSP-4-treated mice $(n=13)$ and control mice $(n=15)$ although the mean values were smaller in the former group. $\boldsymbol{b}$, In the open field, the number and percentage of central square entries were significantly smaller in the DSP-4-treated mice $(n=13)$ compared with the control mice $(n=15)$. The time spent in the central square was not significantly different between groups. The total number of crossings between squares was not different between groups. $c$, In the light-dark box, the time spent in the light box was significantly smaller in the DSP-4-treated mice $(n=13)$ compared with the control mice $(n=15)$. The latency to appear was longer, and the number of transitions was smaller in the DSP-4-treated mice but not significantly. Means \pm SEM. ${ }^{*} p<0.05 ;{ }^{\dagger} p<0.01$ vs saline-injected (control) mice.
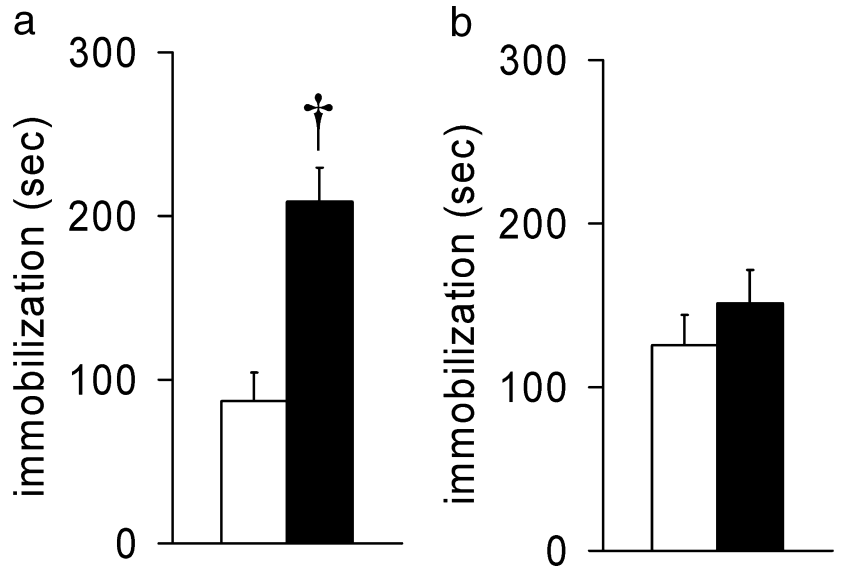

Figure 6. Depression-like behavior in animals injected with either the immunotoxin or DSP-4. $\boldsymbol{a}$, In the immunotoxin-injected mice (closed column, $n=20$ ), the immobilization time was significantly greater compared with the control (sham-operated) mice (open column, $n=$ 15). $\boldsymbol{b}$, In the DSP-4-treated mice (closed column, $n=13$ ), however, the immobilization time was not significantly different compared with the control mice (open column, $n=15$ ). Means \pm SEM. ${ }^{\dagger} p<0.001$ vs control mice. depletion was reached, the ablated LC-NA neurons did not recover following immunotoxin injection, which is in striking contrast to the gradual recovery following DSP-4 (Fritschy and Grzanna, 1992). The long-lasting ablation of the LC-NA neurons may be one of the greatest advantages of the present method, especially for studying the chronic effects of noradrenaline depletion.

The tissue noradrenaline content decreased more profoundly in the immunotoxin-injected group than in the DSP-4-treated group. The difference was remarkable especially in the cerebellum $(95 \%$ [immunotoxin] vs 56\% [DSP-4]) and medulla $(75 \%$ vs $47 \%$ ), although the regional profiles of noradrenaline decrement showed considerable similarity throughout the brain. Tissue dopamine content and serotonin content were not different between the LC-NA neuron-ablated mice and control mice with the exception of some limited brain regions. Interestingly, secondary increases in the dopamine content and serotonin content have ensued in the amygdaloid region. Since the midbrain dopaminergic neurons project directly to the amygdala and amygdaloid dopaminergic receptors are implicated in the modulation of fear and anxiety (Pérez de la Mora et al., 2010; Takahashi et al., 2010), increased dopamine content in the amygdaloid region may be partly involved in the behavioral changes observed in the present study (vide infra).

Following either the immunotoxin-mediated neuronal targeting or DSP-4 treatment, mice presented increases in anxietylike behaviors compared with their respective controls. Although the profiles of behavioral change in the DSP-4-treated mice resembled those in the immunotoxin-treated mice, the effects of ablation appeared more profound in the latter, especially in the EPM and open-field tests. In the light-dark box, the number of transitions was significantly greater in the immunotoxin-treated mice, whereas the time spent in the light box was significantly greater in the DSP-4-treated mice; either of these parameters is considered a measure of increased anxiety. Overall, increased anxiety-like behaviors were observed more consistently throughout the battery following immunotoxin treatment than DSP-4 treatment, and this is in agreement with more prominent decrement of tissue noradrenaline following intervention. The anxiety-like behaviors used in the present study are related to the "novelty response" of rodents (Belzung and Le Pape, 1994), so our results may be consistent with the observations in earlier rat studies, in which "neophobia-like behaviors" were reported following 6-OHDA-induced ablation of the NA system (Mason and Fibiger, 1979; Britton et al., 1984).

Marked increase in depression-like behavior was observed in mice whose LC-NA neurons were ablated by immunotoxin in the present study. In contrast, DSP-4-treated mice did not show any clear increase in depression-like behavior. These results parallel the magnitude of tissue noradrenaline decrease following intervention, suggesting that the increased depression-like behavior may have resulted from the more profoundly reduced tissue noradrenaline in the immunotoxin-treated mice, although any superimposed effect of surgical intervention could not be ruled out. The present results are at variance with the phenotypes of DBH knock-out mice, in which neither increased anxiety-like behavior nor depression-like behavior was observed (Cryan et al., 2001; Marino et al., 2005). The reason for this discrepancy is not clear, but in DBH knock-out mice, noradrenaline is depleted in all the NA nuclei throughout development. This may have resulted in responses different from those in the present study, in which deprivation was made very selectively and only in adulthood. 
Profound loss of LC-NA neurons is characteristic for patients with neurological disorders, including Parkinson's disease and Alzheimer's disease (Benarroch, 2009). It has been hypothesized that degeneration of the LC may be an early event that precedes degeneration of the substantia nigra pars compacta in Parkinson's disease (German et al., 1992). Furthermore, the LC may possibly be involved in both the motor (Gesi et al., 2000; Rommelfanger and Weinshenker, 2007) and nonmotor (Gesi et al., 2000) symptoms of Parkinson's disease. Anxiety and depression are frequently observed nonmotor symptoms of Parkinson's disease (Martínez-Martín and Damián, 2010; Nègre-Pagès et al., 2010), and the present results raise the possibility that the mechanisms of anxiety and/or depression in Parkinsonism could partly be explained by the loss of LC-NA neurons. Because of the remarkable thoroughness and spatial selectivity for targeting the LC-NA neurons, the present mouse model may provide a means for further unraveling the pathological implications of the LC.

\section{References}

Aston-Jones G, Delfs JM, Druhan J, Zhu Y (1999) The bed nucleus of the stria terminalis. A target site for noradrenergic actions in opiate withdrawal. Ann NY Acad Sci 877:486-498.

Aston-Jones G, Iba M, Clayton E, Rajkowski J, Cohen J (2007) The locus coeruleus and regulation of behavioral flexibility and attention: clinical implications. In: Brain norepinephrine (Ordway GA, Schwartz MA, Frazer A, eds), pp 196-235. Cambridge: Cambridge UP.

Belzung C, Le Pape G (1994) Comparison of different behavioral test situations used in psychopharmacology for measurement of anxiety. Physiol Behav 56:623-628.

Benarroch EE (2009) The locus ceruleus norepinephrine system: functional organization and potential clinical significance. Neurology 73:1699-1704.

Berridge CW, Waterhouse BD (2003) The locus coeruleus-noradrenergic system: modulation of behavioral state and state-dependent cognitive processes. Brain Res Rev 42:33-84.

Britton DR, Ksir C, Britton KT, Young D, Koob GF (1984) Brain norepinephrine depleting lesions selectively enhance behavioral responsiveness to novelty. Physiol Behav 33:473-478.

Brunello N, Blier P, Judd LL, Mendlewicz J, Nelson CJ, Souery D, Zohar J, Racagni G (2003) Noradrenaline in mood and anxiety disorders: basic and clinical studies. Int Clin Psychopharmacol 18:191-202.

Chaudhary VK, Queen C, Junghans RP, Waldmann TA, FitzGerald DJ, Pastan I (1989) A recombinant immunotoxin consisting of two antibody variable domains fused to Pseudomonas exotoxin. Nature 339:394-397.

Cryan JF, Dalvi A, Jin SH, Hirsch BR, Lucki I, Thomas SA (2001) Use of dopamine-beta-hydroxylase-deficient mice to determine the role of norepinephrine in the mechanism of action of antidepressant drugs. J Pharmacol Exp Ther 298:651-657.

Cunningham ET, Sawchenko PE (1988) Anatomical specificity of noradrenergic inputs to the paraventricular and supraoptic nuclei of the rat hypothalamus. J Comp Neurol 274:60-76.

Engelbrecht AH, Russell V, Carstens ME, De Villiers AS, Searson A, Jaffer A, Taljaard JJ (1994) Evidence that noradrenergic neurons in the Al and A2 nuclei are lesioned by low doses of 6-OHDA injected into the locus coeruleus. J Neurosci Methods 52:57-60.

España RA, Berridge CW (2006) Organization of noradrenergic efferents to arousal-related basal forebrain structures. J Comp Neurol 496:668-683.

Fritschy J-M, Grzanna R (1989) Immunohistochemical analysis of the neurotoxic effects of DSP-4 identifies two populations of noradrenergic axon terminals. Neuroscience 30:181-197.

Fritschy J-M, Grzanna R (1992) Restoration of ascending noradrenergic projections by residual locus coeruleus neurons: compensatory response to neurotoxin-induced cell death in the adult rat brain. J Comp Neurol 321:421-441.

Gaillet S, Alonso G, Le Borgne R, Barbanel G, Malaval F, Assenmacher I, Szafarczyk A (1993) Effects of discrete lesions in the ventral noradrenergic ascending bundle on the corticotropic stress response depend on the site of the lesion and on the plasma levels of adrenal steroids. Neuroendocrinology 58:408-419.

German DC, Manaye KF, White CL 3rd, Woodward DJ, McIntire DD, Smith WK, Kalaria RN, Mann DM (1992) Disease-specific patterns of locus coeruleus cell loss. Ann Neurol 32:667-676.

Gesi M, Soldani P, Giorgi FS, Santinami A, Bonaccorsi I, Fornai F (2000) The role of the locus coeruleus in the development of Parkinson's disease. Neurosci Biobehav Rev 24:655-668.

González MM, Aston-Jones G (2006) Circadian regulation of arousal: role of the noradrenergic locus coeruleus system and light exposure. Sleep 29:1327-1336.

Jonsson G, Hallman H, Ponzio F, Ross S (1981) DSP4 ( $N$-(2-chloroethyl)$\mathrm{N}$-ethyl-2-bromobenzylamine) - a useful denervation tool for central and peripheral noradrenaline neurons. Eur J Pharmacol 72:173-188.

Keppler-Hafkemeyer A, Brinkmann U, Pastan I (1998) Role of caspases in immunotoxin-induced apoptosis of cancer cells. Biochemistry 37:16934-16942.

Kobayashi K, Morita S, Sawada H, Mizuguchi T, Yamada K, Nagatsu I, Fujita K, Kreitman RJ, Pastan I, Nagatsu T (1995) Immunotoxin-mediated conditional disruption of specific neurons in transgenic mice. Proc Natl Acad Sci U S A 92:1132-1136.

Kreitman RJ, Bailon P, Chaudhary VK, FitzGerald DJP, Pastan I (1994) Recombinant immunotoxins containing anti-Tac $(\mathrm{Fv})$ and derivertives of Pseudomonas exotoxin produce complete regression in mice of an interleukin-2 receptor-expressing human carcinoma. Blood 83:426-434.

Kromer LF, Moore RY (1980) Norepinephrine innervation of the cochlear nuclei by locus coeruleus neurons in the rat. Anat Embryol 158:227-244.

Levitt P, Moore RY (1979) Origin and organization of brainstem catecholamine innervation in the rat. J Comp Neurol 186:505-528.

Logue MP, Growdon JH, Coviella ILG, Wurtman RJ (1985) Differential effects of DSP-4 administration on regional brain norepinephrine turnover in rats. Life Sci 37:403-409.

Marino MD, Bourdélat-Parks BN, Cameron Liles L, Weinshenker D (2005) Genetic reduction of noradrenergic function alters social memory and reduces aggression in mice. Behav Brain Res 161:197-203.

Martínez-Martín P, Damián J (2010) Parkinson disease: depression and anxiety in Parkinson disease. Nat Rev Neurol 6:243-245.

Mason ST, Fibiger HC (1979) Current concepts. I. Anxiety: the locus coeruleus disconnection. Life Sci 25:2141-2147.

Moore RY, Card JP (1984) Noradrenaline-containing neuron systems. In: Handbook of chemical neuroanatomy (Björklund A, Hökfelt T, eds), pp 123-156. Amsterdam: Elsevier.

Murchison CF, Zhang XY, Zhang WP, Ouyang M, Lee A, Thomas SA (2004) A distinct role for norepinephrine in memory retrieval. Cell 117:131-143.

Nègre-Pagès L, Grandjean H, Lapeyre-Mestre M, Montastruc JL, Fourrier A, Lépine JP, Rascol O; DoPaMiP Study Group (2010) Anxious and depressive symptoms in Parkinson's disease: the French cross-sectional DoPaMiP study. Mov Disord 25:157-166.

Palkovits M (1981) Catecholamines in the hypothalamus: an anatomical review. Neuroendocrinology 33:123-128.

Pérez de la Mora M, Gallegos-Cari A, Arizmendi-García Y, Marcellino D, Fuxe K (2010) Role of dopamine receptor mechanisms in the amygdaloid modulation of fear and anxiety: structural and functional analysis. Prog Neurobiol 90:198-216.

Rommelfanger KS, Weinshenker D (2007) Norepinephrine: the redheaded stepchild of Parkinson's disease. Biochem Pharmacol 74:177-190.

Sterpenich V, D’Argembeau A, Desseilles M, Balteau E, Albouy G, Vandewalle G, Degueldre C, Luxen A, Collette F, Maquet P (2006) The locus ceruleus is involved in the successful retrieval of emotional memories in humans. J Neurosci 26:7416-7423.

Takahashi H, Takano H, Kodaka F, Arakawa R, Yamada M, Otsuka T, Hirano Y, Kikyo H, Okubo Y, Kato M, Obata T, Ito H, Suhara T (2010) Contribution of dopamine $\mathrm{D} 1$ and $\mathrm{D} 2$ receptors to amygdala activity in human. J Neurosci 30:3043-3047.

Yanai K, Son LZ, Endou M, Sakurai E, Nakagawasai O, Tadano T, Kisara K, Inoue I, Watanabe T, Watanabe T (1998) Behavioural characterization and amounts of brain monoamines and their metabolites in mice lacking histamine H1 receptors. Neuroscience 87:479-487. 